\title{
Advantages of CyberKnife for inoperable stage I peripheral non-small-cell lung cancer compared to three-dimensional conformal radiotherapy
}

\author{
AN-NA TONG ${ }^{1 *}$, PENG YAN ${ }^{2 *}$, GUANG-HUI YUAN ${ }^{1}$, XIAO-YAN LV ${ }^{1}$, \\ $\mathrm{HAI} \mathrm{GONG}^{1}$, HUI ZHAO ${ }^{1}$ and YAN-MING WANG ${ }^{1}$ \\ ${ }^{1}$ Department of Radiation Oncology, Jinan Military General Hospital, Jinan, Shandong 250031; \\ ${ }^{2}$ Department of Oncology, Jinan Central Hospital Affiliated to Shandong University, \\ Jinan, Shandong 250013, P.R. China
}

Received October 2, 2014; Accepted November 25, 2014

DOI: $10.3892 / \mathrm{mco} .2014 .475$

\begin{abstract}
This study was conducted to compare the clinical curative effect and acute radiation lung reactions between CyberKnife (CK) and three-dimensional conformal radiotherapy (3DCRT) treatment for inoperable stage I peripheral non-small-cell lung cancer (NSCLC). We retrospectively analyzed 68 patients with inoperable stage I peripheral NSCLC between 2012 and 2013 in our institution. The CK patients were treated with $42-60 \mathrm{~Gy}$ in three fractions, while the 3DCRT patients were treated with a total of $60 \mathrm{~Gy}$, at $2 \mathrm{~Gy}$ per fraction. The patients were followed up and the clinical outcome was evaluated according to the Response Evaluation Criteria in Solid Tumours. We assessed the presence of acute radiation pneumonitis and pulmonary function status by thoracic scan and pulmonary function tests following CK and 3DCRT treatment. The binary univariate logistic regression analysis demonstrated that treatment method and forced expiratory volume in $1 \mathrm{sec} /$ forced vital capacity (FEV1/FVC) prior to treatment (pre-FEV1/FVC) were the main factors affecting the risk of radiation pneumonitis. The analysis of these factors through multivariate logistic regression method demonstrated that treatment method for grade 1 and 2 [odds ratio $(\mathrm{OR})=7.866$ and 11.334 , respectively) and pre-FEV1/FVC for grade 1, 2 and $3(\mathrm{OR}=5.062,11.498$ and 15.042, respectively) were significant factors affecting the risk of radiation pneumonitis $(\mathrm{P}<0.05)$. The 68 patients were divided into two subgroups using the threshold of pre-FEV1/FVC selected by the receiver operating characteristic curve. There were
\end{abstract}

Correspondence to: Professor Yan-Ming Wang, Department of Radiation Oncology, Jinan Military General Hospital, 25 Normal School Road, Tianqiao District, Jinan, Shandong 250031, P.R. China E-mail: ymwang64@163.com

${ }^{*}$ Contributed equally

Key words: CyberKnife, three-dimensional conformal radiotherapy, non-small-cell lung cancer, radiation pneumonitis significant differences between the 3DCRT and CK treatment in both the pre-FEV1/FVC $<68 \%$ and $\geq 68 \%$ subgroups for radiation pneumonitis ( $\mathrm{P}=0.023$ and 0.002 , respectively). There was no statistically significant change in FVC, FEV1 and carbon monoxide diffusion capacity (DCLO) in the CK group, whereas there was a decrease in DCLO in the 3DCRT group. The complete remission rate was 40 vs. $34.2 \%$ at 1 year in the CK and 3DCRT groups, respectively. In conclusion, in this cohort of patients with inoperable stage I peripheral NSCLC, CK appears to be a safe and superior alternative to conventionally fractionated radiotherapy.

\section{Introduction}

Lung cancer is the leading cause of cancer-related mortality. Non-small-cell lung cancer (NSCLC) represents the majority of lung cancer cases. Lobectomy is the standard therapy used for stage I peripheral NSCLC. However, lobectomy is a major surgical procedure that is often associated with a clinically significant decline in pulmonary function. Furthermore, due to the usually high rate of comorbidities and poor baseline pulmonary function, patients with early NSCLC are often considered medically inoperable. Historically, the treatment outcomes of inoperable patients with clinical stage I NSCLC have been poor (1). A large field must be irradiated to account for respiratory tumour motion; as a consequence, conventional or standard radiotherapy for early-stage NSCLC patients usually leads to excessive exposure of normal lung tissue. Three-dimensional conformal radiotherapy (3DCRT) has been widely applied for the treatment of lung malignancies. The lower conformality of 3DCRT results in a larger volume receiving the therapeutic dose, which may be preferable for a moving target. In addition, locoregional control and survival are typically disappointing with conventional radiation therapy. Clinically significant radiation pneumonitis usually develops in $13-37 \%$ of patients receiving radical dose radiotherapy against lung cancer (2).

Over the last few years, stereotactic body radiation therapy (SBRT) has been applied as a standard treatment option in the treatment against inoperable early-stage peripheral 
NSCLC. CyberKnife (CK) is a non-invasive robotic system, which allows delivery of SBRT through precise, nearly real-time image-guided tracking of a mobile target. Previous studies (3-5) demonstrated excellent local control rates of $90-97 \%$ at $2-3$ years' follow-up, with minimal associated toxicities.

There are currently no randomized trials comparing the efficacy and acute toxicity of CK and 3DCRT in thoracic malignancies. The most common acute toxicity is radiation pneumonitis following lung radiotherapy. Our objective was to compare the efficacy and acute toxicity of these two techniques against inoperable stage I peripheral NSCLC.

\section{Materials and methods}

Patient eligibility. We retrospectively investigated a total of 68 patients with pathologically confirmed clinical stage I peripheral NSCLC between 2012 and 2013. A total of 38 patients received 3DCRT with flattened beams, whereas the remaining 30 patients were treated with $\mathrm{CK}$. The main characteristics of the patient cohorts are summarized in Table I. Thoracic computed tomography (CT) imaging and routine pulmonary function tests (PFTs) were performed prior to treatment. The patients were considered ineligible for surgical resection due to advanced age or the presence of comorbidities.

Fiducial placement. All the stage I NSCLC patients were implanted using fiducial markers with the Synchrony ${ }^{\circledR}$ Respiratory Motion Tracking system (6). Under conscious sedation and local anesthesia, 3-5 gold fiducials measuring $0.8 \mathrm{~mm}$ in diameter by $3 \mathrm{~mm}$ in length (Civco Medical Solutions, Orange City, IA, USA) were placed with adequate spacing $(1-2 \mathrm{~cm})$ in or near tumours under CT guidance $(7,8)$.

Treatment planning. We treated a group of patients with the CyberKnife frameless robotic radiosurgery system (Accuray, Inc., Sunnyvale, CA, USA). We obtained fine-cut (1.5-mm) treatment planning CTs 7-10 days following fiducial placement during a full-inhalation breath-hold. Gross tumour volume (GTV) was contoured with lung windows. The GTV margin was expanded by $5 \mathrm{~mm}$ to set the planning treatment volume (PTV). All the critical thoracic structures and the lungs were contoured to ensure that incidental radiation delivered to these structures was limited according to the reports of the American Association of Physicists in Medicine Task Group 101. A treatment plan from MultiPlan software (Accuray, Inc.) was made using the CyberKnife non-isocentric, inverse-planning ray-tracing algorithm with tissue density heterogeneity corrections for lung. Lower doses within the range of 42-60 Gy in three fractions were prescribed when concerns regarding adjacent critical structures arose and when patients were considered to exhibit severe pulmonary dysfunction. The biologically effective dose (BED) was 100.8-180 Gy for patients undergoing CK treatment. The radiation dose was prescribed to an isodose line that covered $\geq 95 \%$ of the PTV and caused the 30 -Gy isodose contour to extend a minimum of $1 \mathrm{~cm}$ from the GTV. The percentage of the total lung volume receiving $\geq 15 \mathrm{~Gy}$ (V15) was limited to $15 \%$.

Radiation was delivered with photon beams of $6 \mathrm{MV}$ from a linear accelerator (Elekta Precise; Elekta Oncology Systems,
Table I. Patient baseline characteristics.

\begin{tabular}{|c|c|c|c|c|}
\hline \multirow[b]{2}{*}{ Variables } & \multirow{2}{*}{$\begin{array}{l}\text { Entire } \\
\text { cohort } \\
(n=68)\end{array}$} & \multicolumn{2}{|c|}{ Treatment groups } & \multirow[b]{2}{*}{ P-value } \\
\hline & & $\begin{array}{c}\text { 3DCRT } \\
(n=38)\end{array}$ & $\begin{array}{c}\text { CK } \\
(n=30)\end{array}$ & \\
\hline Age (years) & & & & 0.919 \\
\hline$<70$ & 29 & 16 & 13 & \\
\hline$\geq 70$ & 39 & 22 & 17 & \\
\hline Gender & & & & 0.243 \\
\hline Male & 40 & 20 & 20 & \\
\hline Female & 28 & 18 & 10 & \\
\hline ECOG PS & & & & 0.340 \\
\hline $0-1$ & 41 & 21 & 20 & \\
\hline 2 & 27 & 17 & 10 & \\
\hline Smoking & & & & 0.174 \\
\hline Yes & 38 & 24 & 14 & \\
\hline No & 30 & 14 & 16 & \\
\hline Pre-FEV1/FVC & & & & 0.660 \\
\hline$<68 \%$ & 23 & 12 & 11 & \\
\hline$\geq 68 \%$ & 45 & 26 & 19 & \\
\hline Tumor location & & & & 0.697 \\
\hline Upper & 47 & 27 & 20 & \\
\hline Lower & 21 & 11 & 10 & \\
\hline Clinical stage & & & & 0.382 \\
\hline T1N0M0 & 24 & 12 & 12 & \\
\hline T2aN0M0 & 44 & 26 & 18 & \\
\hline Pathology of NSCLC & & & & 0.265 \\
\hline Squamous & 20 & 11 & 9 & \\
\hline Adenocarcinoma & 40 & 20 & 20 & \\
\hline Other & 8 & 7 & 1 & \\
\hline
\end{tabular}

3DCRT, three-dimensional conformal radiotherapy; CK, CyberKnife; ECOG PS, Eastern Cooperative Oncology Group performance status; FEV1, forced expiratory volume during the first second; FVC, forced vital capacity; NSCLC, non-small-cell lung cancer.

Crawley, UK) in the 3DCRT group. Each of the patients was irradiated for $60 \mathrm{~Gy}, 2 \mathrm{~Gy} /$ fraction, once per day, 5 days per week. The BED was 72 Gy for the patients receiving 3DCRT treatment. Radiation Therapy Planning software (Pinnacle3; Philips Medical Systems, Fitchburg, WI, USA) was used to design the radiation plan. In the 3DCRT plans, due to the unavailability of 4DCT imaging, larger margins were used to define the PTV $(10,10$ and $15 \mathrm{~mm}$ in the latero-lateral, antero-posterior and cranio-caudal directions, respectively) to account for respiratory motion. The lungs, heart and spinal cord were considered as organs at risk (OARs). The planning objective was to cover $95 \%$ of the volume with $95 \%$ of the dose for the PTV. The constraints for the OARs were Dmax $<20$ Gy for the spinal cord and Dmax $<30$ Gy for the heart. For the joint lungs, exclusive of PTV, the following constraints were set: V30Gy $<20 \%$ and a mean lung dose $<4$ Gy.

The BED was calculated with the following linear quadratic formula: $\mathrm{BED}=(\mathrm{nd})[1+\mathrm{d} /(\alpha / \beta)]$. Factor $\alpha / \beta$ was assumed to be 
Table II. Results of univariate logistic regression analysis for the correlation with radiation pneumonitis.

\begin{tabular}{lccccc}
\hline Variables & $\mathrm{B}$ & $\mathrm{SE}$ & Wald & P-value & OR (95\% CI) \\
\hline Age $(<70 / \geq 70$ years) & -0.604 & 0.775 & 0.068 & 0.436 & $0.547(0.120-2.499)$ \\
Gender (male/female) & 0.423 & 1.830 & 0.053 & 0.817 & $1.526(0.042-55.069)$ \\
ECOG PS & & & & & \\
$\quad 1 / 0$ & 1.204 & 0.619 & 3.781 & 0.052 & $3.333(0.991-11.271)$ \\
$2 / 0$ & 0.223 & 0.626 & 0.127 & 0.721 & $1.250(0.367-4.262)$ \\
Smoking (no/yes) & -0.483 & 0.837 & 0.333 & 0.564 & $0.617(0.120-3.182)$ \\
Pathology & & & & & \\
Squamous/other & 0.499 & 1.241 & 0.162 & 0.688 & $1.647(0.145-18.753)$ \\
Adenocarcinoma/other & -0.027 & 1.906 & 0.000 & 0.989 & $0.973(0.023-40.758)$ \\
Tumor location (upper/lower) & -0.149 & 0.723 & 0.042 & 0.837 & $0.862(0.209-3.554)$ \\
Clinical stage (T1/T2a) & -0.845 & 0.725 & 1.358 & 0.244 & $0.430(0.104-1.778)$ \\
Treatment method (3DCRT/CK) & 2.538 & 0.797 & 10.148 & $0.001^{\mathrm{a}}$ & $12.651(2.655-60.285)$ \\
Pre-FEV1/FVC (<68\%/ $\geq 68 \%)$ & 3.082 & 0.913 & 11.398 & $0.001^{\mathrm{a}}$ & $21.793(3.642-130.400)$ \\
Constant & -1.825 & 2.313 & 0.623 & 0.430 & 0.161 \\
\hline
\end{tabular}

${ }^{\mathrm{a}} \mathrm{P}<0.05$. OR, odds ratio; CI, confidence interval; ECOG PS, Eastern Cooperative Oncology Group performance status; 3DCRT, three-dimensional conformal radiotherapy; CK, CyberKnife; FEV1, forced expiratory volume during the first second; FVC, forced vital capacity.

$10 \mathrm{~Gy}$, with the variables $\mathrm{n}$ and $\mathrm{d}$ representing the number of fractions and the dose per fraction, respectively.

Treatment delivery. In brief, pretreatment fluoroscopy confirmed that fiducial motion was associated with tumour motion. The patients were then transferred to the CK suite and laid supine on the treatment table with their arms at their sides. Three red light-emitting diodes (LEDs) were placed above the patient's anterior torso directing toward the camera array. Fiducials were located with orthogonal X-ray imagers. A correlation model was formed between the LEDs tracked continuously by the camera array and the fiducial positions imaged periodically by the X-ray targeting system. During treatment delivery the tumour position was tracked through the live camera array signal and correlation model; the robotic arm moved the linear accelerator to maintain precise alignment with the tumour throughout the respiratory cycle. Fiducials were imaged prior to delivery of every third beam to check targeting accuracy and to update the correlation model.

The patients in the 3DCRT group were placed in a supine position with their hands on their head and fingers interlocked. The body position was fixed and respiratory movement was appropriately limited with a vacuum pad. By planning images transmitted by the network from the three-dimensional treatment planning system, the patients received conventional external radiotherapy.

Follow-up studies. The patients were followed up for 1 year after the date of CK or 3DCRT treatment completion. Clinical outcome was evaluated through physical examination and thoracic CT imaging prior to and after treatment. Toxicities were graded according to the Radiation Therapy Oncology
Group criteria $(9,10)$. Clinical outcome was tumour control defined as disappearance, shrinkage or growth of the tumour, assessed according to the Response Evaluation Criteria in Solid Tumours, which are defined as follows: complete remission (CR), disappearance of all target lesions; partial remission, 30\% decrease in the sum of the longest diameter of target lesions; progressive disease, $20 \%$ increase in the sum of the longest diameter of target lesions; stable disease, small changes that do not meet the abovementioned criteria (11). To asses the effects of radiotherapy on functional tests, routine PFTs were performed at 1, 3, 6 and 12 months, including forced vital capacity (FVC), forced expiratory volume during the first second (FEV1) and carbon monoxide diffusion capacity (DCLO).

Statistical analysis. The correlations between FEV1/FVC prior to treatment (pre-FEV1/FVC) and radiation pneumonitis were evaluated by using Spearman's rank correlation. A receiver operating characteristic (ROC) curve was generated to assess the pre-FEV1/FVC for radiation pneumonitis. The pre-FEV1/FVC values were divided into two groups using the threshold of pre-FEV1/FVC selected by the ROC curve. The differences in categorical variables between the $\mathrm{CK}$ and 3DCRT groups were compared using Chi-square tests. Univariate and multivariate logistic regression analyses were used to indicate the association between radiation pneumonitis and a number of variables, including age, gender, Eastern Cooperative Oncology Group performance status, radiation therapy techniques and pre-FEV1/FVC. The comparison of radiation pneumonitis between the two subgroups (pre-FEV1/FVC $<68 \%$ vs. $\geq 68 \%$ ) with different radiation therapy techniques was assessed using the rank-sum test.

The differences between pre- and post-treatment in terms of PFTs for the two groups were assessed using the t-test for 
Table III. Results of multivariate logistic regression analysis for the correlation with radiation pneumonitis.

\begin{tabular}{|c|c|c|c|c|c|}
\hline Radiation pneumonitis & $\mathrm{B}$ & STD & Wald & P-value & OR $(95 \% \mathrm{CI})$ \\
\hline \multicolumn{6}{|l|}{ Grade 1} \\
\hline Intercept & -2.291 & 0.660 & 12.052 & 0.001 & \\
\hline Pre-FEV1/FVC $(<68 \% / \geq 68 \%)$ & 1.622 & 0.729 & 4.955 & 0.026 & $5.062(1.214-21.113)$ \\
\hline 3DCRT/CK & 2.063 & 0.717 & 8.276 & 0.004 & $7.866(1.930-32.066)$ \\
\hline \multicolumn{6}{|l|}{ Grade 2} \\
\hline Intercept & -3.516 & 0.955 & 13.566 & 0.000 & \\
\hline Pre-FEV1/FVC $(<68 \% / \geq 68 \%)$ & 2.442 & 0.853 & 8.206 & 0.004 & $11.498(2.163-61.134)$ \\
\hline 3DCRT/CK & 2.428 & 0.940 & 6.673 & 0.010 & $11.334(1.796-71.517)$ \\
\hline \multicolumn{6}{|l|}{ Grade 3} \\
\hline Intercept & -21.846 & 0.839 & 678.731 & 0.000 & \\
\hline $\begin{array}{l}\text { Pre-FEV1/FVC }(<68 \% / \geq 68 \%) \\
3 \text { DCRT/CK }{ }^{\mathrm{a}}\end{array}$ & 2.711 & 1.399 & 3.752 & 0.050 & $15.042(0.969-233.605)$ \\
\hline
\end{tabular}

${ }^{a}$ No patients in the CK group had grade 3 radiation pneumonitis (the OR was infinite). The reference category is 0 . This parameter is set to zero, as it is redundant. OR, odds ratio; CI, confidence interval; FEV1, forced expiratory volume during the first second; FVC, forced vital capacity; 3DCRT, three-dimensional conformal radiotherapy; CK, CyberKnife.

statistics. All the analyses were performed using the SPSS 13.0 software (SPSS Inc., Chicago, IL, USA). P $<0.05$ was considered to indicate a statistically significant difference.

\section{Results}

Patients. A total of 68 consecutive patients diagnosed with NSCLC were treated with definitive radiation between 2012 and 2013, with 38 patients receiving 3DCRT and 30 receiving CK. Among the patients treated with 3DCRT, 20 (53\%) were men and $18(47 \%)$ women, with a median age of 75.7 years (range, 65-82 years). Among the patients treated with CK, $20(67 \%)$ were men and $10(33 \%)$ women, with a median age of 74.7 years (range, 66-83 years). There was no statistically significant difference between the two treatment groups regarding patient characteristics (Table I).

Correlation of different variables with radiation pneumonitis. A positive correlation was found between pre-FEV1/FVC and radiation pneumonitis $(\mathrm{r}=0.844, \mathrm{P}=0.000)$. We divided the 68 patients into two subgroups using the threshold of pre-FEV1/FVC selected by the ROC curve. The binary univariate logistic regression analysis revealed a significant association of radiation pneumonitis with treatment method (3DCRT vs. CK) and pre-FEV1/FVC (OR=12.651 and 21.793, respectively) (Table II). The analysis of these factors by the multivariate logistic regression method demonstrated that treatment method for grades 1 and $2(\mathrm{OR}=7.866$ and 11.334, respectively) and pre-FEV1/FVC for grades 1,2 and 3 $(\mathrm{OR}=5.062,11.498$ and 15.042, respectively) were significant factors affecting the risk of radiation pneumonitis $\mathrm{P}<0.05$ ) (Table III), with an increased risk for pre-FEV1/FVC $<68 \%$ compared to pre-FEV1/FVC $\geq 68 \%$ and in the 3DCRT compared to the CK group. There were 3 patients in the 3DCRT group with grade 3 radiation pneumonitis, whereas there were none in the CK group (the OR was infinite).
Table IV. Comparison of different grades of radiation pneumonitis between 3DCRT and CK treatment in two subgroups (pre-FEV1/FVC $<68 \%$ and $\geq 68 \%$ ).

\begin{tabular}{lccccc}
\hline & \multicolumn{2}{c}{$\begin{array}{c}\text { Pre-FEV1/FVC } \\
(<68 \%)\end{array}$} & & \multicolumn{2}{c}{$\begin{array}{c}\text { Pre-FEV1/FVC } \\
(\geq 68 \%)\end{array}$} \\
\cline { 2 - 3 } \cline { 5 - 6 } Grade & 3 DCRT & CK & & 3 3CRT & CK \\
\hline 0 & 1 & 5 & & 14 & 19 \\
1 & 3 & 3 & & 9 & 1 \\
2 & 5 & 3 & & 3 & 0 \\
3 & 3 & 0 & & 0 & 0 \\
Mean rank & 15.04 & 8.68 & & 27.67 & 18.08 \\
P-value & 0.023 & 0.002 & & \\
\hline
\end{tabular}

3DCRT, three-dimensional conformal radiotherapy; CK, CyberKnife; FEV1, forced expiratory volume during the first second; FVC, forced vital capacity.

Comparison of radiation pneumonitis grades between $3 D C R T$ and $C K$ in the pre-FEVI/FVC $<68 \%$ and $\geq 68 \%$ subgroups. In pre-FEV1/FVC $<68 \%$ and $\geq 68 \%$ groups, the grades of radiation pneumonitis with $\mathrm{CK}$ treatment were lower compared to those with 3DCRT treatment (mean rank, 8.68 vs. 15.04 and 18.08 vs. 27.67 , respectively). There were significant differences between the 3DCRT and CK treatment in the pre-FEV1/FVC $<68 \%$ and $\geq 68 \%$ subgroups $(\mathrm{P}=0.023$ and 0.002) (Table IV).

Comparison of pulmonary function tests between 3DCRT and $C K$. Considering the effects of radiotherapy on functional tests, there were no significant differences in parameters such as FVC and FEV1 at pre- and post-treatment (1, 3, 6 and 12 months) in the 3DCRT group. However, there were 
Table V. Response to treatment on computed tomography morphological scan.

\begin{tabular}{|c|c|c|c|c|c|c|}
\hline \multirow[b]{2}{*}{ Response } & \multicolumn{3}{|c|}{ 3DCRT, no. (\%) } & \multicolumn{3}{|c|}{ CK, no. (\%) } \\
\hline & 3 months & 6 months & 12 months & 3 months & 6 months & 12 months \\
\hline Complete remission & $9(23.7 \%)$ & $13(34.2 \%)$ & $13(34.2 \%)$ & $10(33.3 \%)$ & $12(40.0 \%)$ & $12(40.0 \%)$ \\
\hline Partial remission & $15(39.5 \%)$ & $17(44.7 \%)$ & $14(36.8 \%)$ & $12(40.0 \%)$ & $13(43.3 \%)$ & $7(23.3 \%)$ \\
\hline Stable disease & $13(34.2 \%)$ & $8(21.1 \%)$ & $11(29.0 \%)$ & $8(26.7 \%)$ & $5(16.7 \%)$ & $11(36.7 \%)$ \\
\hline Progressive disease & $1(2.6 \%)^{\mathrm{a}}$ & $0(0.0 \%)$ & $0(0.0 \%)$ & $0(0.0 \%)$ & $0(0.0 \%)$ & $0(0.0 \%)$ \\
\hline
\end{tabular}

${ }^{a}$ One patient exhibited disease progression at 3 months and underwent chemotherapy; the patient then achieved partial remission at 6 months and stable disease at 12 months. 3DCRT, three-dimensional conformal radiotherapy; CK, CyberKnife.
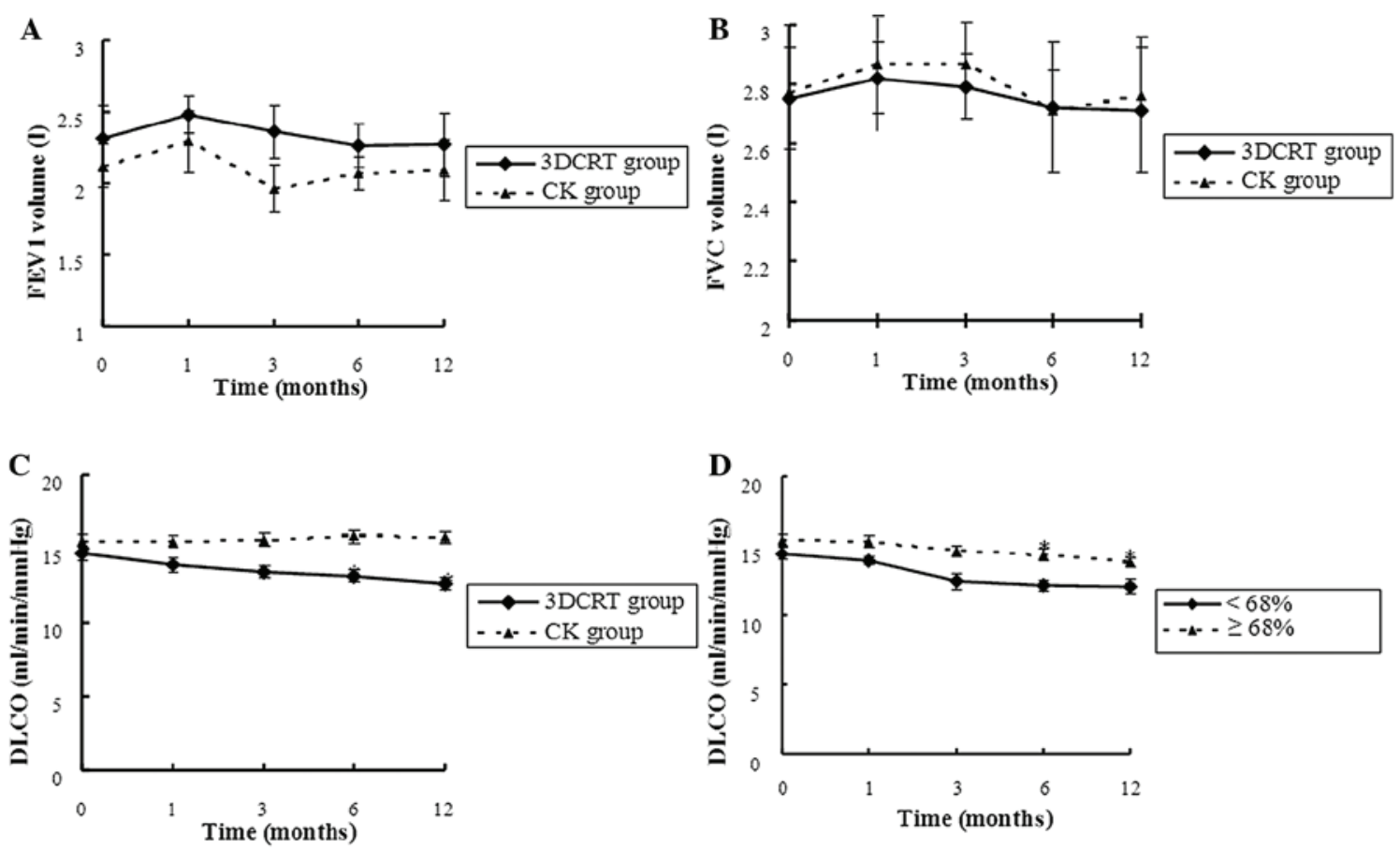

Figure 1. Comparison of pulmonary function tests following 3DCRT and CK treatment. Differences in (A) FEV1, (B) FVC and (C) DLCO pre- and post-treatment (1, 3, 6 and 12 months) in the 3DCRT and CK groups. (D) Differences in DLCO at pre- and post-treatment (1,3, 6 and 12 months) in the two subgroups (pre-FEV1/FVC $<68$ and $\geq 68 \%$ ) of the 3DCRT group. "The follow-up results were compared to the values prior to treatment (P<0.05). 3DCRT, three-dimensional conformal radiotherapy; CK, CyberKnife; FEV1, forced expiratory volume during the first second; FVC, forced vital capacity; DLCO, carbon monoxide diffusion capacity.

statistically significant decreases in the mean DLCO in the 3DCRT group at 6 and 12 months post-treatment. Additionally, there was a distinct decrease in the mean DLCO in the two subgroups (pre-FEV1/FVC $<68 \%$ and $\geq 68 \%$ ) of the 3DCRT group at 6 and 12 months. In the CK group, there was no significant change in FVC, FEV1 or DCLO (Fig. 1).

All the patients underwent follow-up evaluation at 3,6 and 12 months. No patients were lost to follow-up and no patient succumbed to the disease within that time period. The local control rate at 1 year was $100 \%$ in patients treated with $\mathrm{CK}$ and $97.4 \%$ in those treated with 3DCRT. The follow-up results at 6 months were compared to those at 3 months; and the follow-up results at 12 months were compared to those at 6 months.

Response to treatment on CT scan. As shown in Table V, the major radiological response (CR), as evaluated by CT scan at 3, 6 and 12 months, was recorded in 9/38 (23.7\%) vs. 10/38 (33.3\%), $13 / 38(34.2 \%)$ vs. $12 / 30(40.0 \%)$ and $13 / 38(34.2 \%)$ vs. $12 / 30$ $(40.0 \%)$ of patients treated with 3DCRT vs. CK, respectively.

\section{Discussion}

CK is a new type of SBRT, which is able to deliver radiation from more angles utilizing image guidance and real-time target tracking. CK treatment has been shown to be very effective for stage I NSCLC in several studies published over the last few years $(3,4,6,12,13)$.

Numerous studies demonstrated that an increase in radiological doses may increase the tumour control rates (14). However, further dose escalation with broad-field radiotherapy may induce higher toxicity, as healthy tissues also receive a higher dose of radiation, which decreases the patients' survival rates (15). 
The binary univariate and multivariate logistic regression analyses demonstrated that the risk of radiation pneumonitis is associated with treatment methods and pre-FEV1/FVC. Patients with underlying lung diseases may exhibit poor lung function, lower-than-normal FVC and FEV1, insufficient respiratory ventilation and air exchange function and low lung compliance; thus, such patients are more likely susceptible to radiation pneumonitis, as reported by a number of experimental studies (15-17). In all the patients, the risk and grade of radiation pneumonitis were higher in the 3DCRT compared to those in the CK group. In the ROC curve of the pre-FEV1/FVC of patients who were divided into two subgroups, the threshold was set at $\mathrm{FEV} 1 / \mathrm{FVC}=68 \%$. In the two groups, the incidence of radiation pneumonitis in the 3DCRT group was significantly higher compared to that in the CK group. Several studies have established an association between the risk of radiation pneumonitis and dose-volume distribution in the lung (18). CK appeared to be an appropriate option for further increasing the tumour biological equivalent dose, without increasing normal tissue toxicity.

In our study, we compared these two radiotherapy approaches according to lung function changes prior to and following radiotherapy. When all the patients were evaluated at 1, 3, 6 and 12 months, no significant changes in FVC and FEV1 were observed, which was possibly due to offsetting between radiation-induced lung tissue damage and tumour regression-induced ventilation function improvement. In the 3DCRT group, DLCO (a measure of diffusion capacity) was significantly decreased; such a tendency was not observed in the CK group. A previous study by Lopez et al (19) indicated that the percentage of the decrease in DLCO is closely associated with severe radiation pneumonitis.

In lung cancer radiotherapy, pulmonary surfactant is released into the alveolar cavity, leading to changes in alveolar tension, alveolar collapse and atelectasis. Furthermore, the injury of type II alveolar epithelial cells and hyperplasia of the collagen fibers lead to alveolar wall and capillary endothelial cell thickening. In addition, edematous fluid within the cell layer leads to the increased diffusion distance and impaired diffusion.

CK successfully preserved the lung diffusion capacity, as measured by PFTs. Our 3DCRT treatment group exhibited a higher risk of radiation pneumonitis compared to that in the CK group, which may be associated with the lower radiation doses to normal tissues in the CK group and the reduced effect of these low doses on diffusion function.

We evaluated the therapeutic effect following radiotherapy through CT scan at different time points. The local tumour control rate in the 3DCRT group reached $97.4 \%$, while that of the CK group reached $100 \%$. The CR rates in the CK group at 3, 6 and 12 months were higher compared to those in the 3DCRT group, as higher doses for tumour target volumes led to higher local tumour control rates. The commonly accepted dose scheme of $60 \mathrm{~Gy}$ in three fractions for peripheral lung tumours, which is equivalent to $150 \mathrm{~Gy}$ in $2 \mathrm{~Gy}$ fractions if $\alpha / \beta$ is $10 \mathrm{~Gy}$ (normalised total dose), was adopted as our standard. When compared to 3DCRT, the average exposure dose increased by $140 \%$, significantly boosting the biological doses, but with small exposure scopes for normal tissues (20).

The mechanism accounting for higher biological doses via $\mathrm{CK}$ and its low exposure dose targeting normal tissues is as follows: the delivery of hundreds of radiation beams while continuously tracking and compensating for respiratory tumour motion ensures that the gross tumour and radial microscopic extension are effectively treated. The higher the number of spread-out non-coplanar beams, the more likely the system is to form a rapid dose fall-off (steep dose gradient). The device used in this study addresses the challenge of respiratory motion using an image-guided real-time targeting/tracking system, resulting in a significantly reduced targeting uncertainty. In addition, a protracted fractionation schedule, which is often used to minimise the risk of radiotherapy-related pneumonitis, may result in poorer outcomes due to accelerated tumour cell repopulation during the radiation course $(21,22)$. Precise radiotherapy by using $\mathrm{CK}$ uses high single doses and few segmentation numbers, together with several advantages that are absent in conventional radiotherapy. For example, i) $\mathrm{CK}$ treatment does not require any method to limit breathing due to tumour tracking; and ii) CK requires shorter hospitalization time and is associated with tolerable adverse reactions, thus it is easier to obtain the patients' consent. In our study, CK was proven to be feasible and safe, while achieving excellent rates of local disease control with limited toxicity to surrounding tissues and, in several cases, may be curative for patients who are not candidates for surgery. In experiments performed by other research centers, CK achieved higher locoregional control and survival rates similar to wedge resection (23).

$\mathrm{CK}$ has shown favorable prospects in stereotactic radiosurgery. However, several problems regarding CK require further investigation: i) although the administered exposure doses and segmentation methods are currently based on biological equivalent doses, the use of $\mathrm{CK}$ is, to a great extent, experience-oriented and, in several recent literature reports, long-term follow-up results supporting the effects of these dose modes on partial tumour recurrence and data on future complications for large-sample cases are not available; and ii) a significant number of extracranial tumours require implanting metal particle markers around the tumours, which may be associated with certain complications.

\section{Acknowledgements}

This study was supported by a grant from the China Postdoctoral Science Foundation (no. 20080431411).

\section{References}

1. Qiao X, Tullgren O, Lax I, et al: The role of radiotherapy in treatment of stage I non-small cell lung cancer. Lung Cancer 41: $1-11,2003$.

2. Rodrigues G, Lock M, D'Souza D, et al: Prediction of radiation pneumonitis by dose-volume histogram parameters in lung cancer - a systematic review. Radiother Oncol 71: 127-138, 2004.

3. Brown WT, Wu X, Fayad F, et al: CyberKnife radiosurgery for stage I lung cancer: results at 36 months. Clin Lung Cancer 8: 488-492, 2007.

4. van der Voort van Zyp NC, Prévost JB, Hoogeman MS, et al: Stereotactic radiotherapy with real-time tumor tracking for non-small cell lung cancer: clinical outcome. Radiother Oncol 91: 296-300, 2009.

5. Timmerman R, Paulus R, Galvin J, et al: Stereotactic body radiation therapy for inoperable early stage lung cancer. JAMA 303: 1070-1076, 2010.

6. Collins BT, Vahdat S, Erickson K, et al: Radical cyberknife radiosurgery with tumor tracking: an effective treatment for inoperable small peripheral stage I non-small cell lung cancer. J Hematol Oncol 2: 1, 2009. 
7. Banovac F, McRae D, Dieterich S, et al: Percutaneous placement of fiducial markers for thoracic malignancies. In: Robotic Radiosurgery: Treating Tumors that Move with Respiration. Urschel HC, Kresel JJ, Luketich JD, Papiez L and Timmerman RD (eds). Springer-Verlag, Berlin, pp15-29, 2007.

8. Yousefi S, Collins BT, Reichner CA, et al: Complications of thoracic computed tomography-guided fiducial placement for the purpose of stereotactic body radiation therapy. Clin Lung Cancer 8: 252-256, 2007.

9. Radiation Therary Oncology Group: Acute radiation morbidity scoring criteria. http://www.rtog.org/ResearchAssociates/ AdverseEventReporting/AcuteRadiationMorbidityScoringCriteria. aspx. Accessed May 1,2012.

10. Radiation Therapy Oncology Group/European Organization for Research and Treatment of Cancer: Late radiation morbidity scoring schema. Available at: http://www.rtog.org/Research Associates/AdverseEventReporting/RTOGEORTCLate RadiationMorbidityScoringSchema.aspx. Accessed May 1, 2012.

11. Therasse P, Arbuck SG, Eisenhauer EA, et al: New guidelines to evaluate the response to treatment in solid tumors. European Organization for Research and Treatment of Cancer, National Cancer Institute of the United States, National Cancer Institute of Canada. J Natl Cancer Inst 92: 205-216, 2000.

12. Vahdat S, Oermann EK, Collins SP, et al: CyberKnife radiosurgery for inoperable stage IA non-small cell lung cancer: ${ }^{18} \mathrm{~F}$-fluorodeoxyglucose positron emission tomography/computed tomography serial tumor response assessment. J Hemato Oncol 3: 6, 2010.

13. Collins BT, Erickson K, Reichner CA, et al: Radical stereotactic radiosurgery with real-time tumor motion tracking in the treatment of small peripheral lung tumors. Radiat Oncol 2: 39, 2007.

14. Mehta M, Scrimger R, Mackie R, et al: A new approach to dose escalation in non-small-cell lung cancer. Int J Radiat Oncol Biol Phys 49: 23-33, 2001.
15. Robnett TJ, Machtay M, Vines EF, et al: Factors predicting severe radiation pneumonitis in patients receiving definitive chemoradiation for lung cancer. Int J Radiat Oncol Biol Phys 48: 89-94, 2000.

16. Kocak Z, Borst GR, Zeng J, et al: Prospective assessment of dosimetric/physiologic-based models for predicting radiation pneumonitis. Int J Radiat Oncol Biol Phys 67: 178-186, 2007.

17. Lind PA, Marks LB, Hollis D, et al: Receiver operating characteristic curves to assess predictors of radiation-induced symptomatic lung injury. Int J Radiat Oncol Biol Phys 54: 340-347, 2002.

18. Marks LB, Bentzen SM, Deasy JO, et al: Radiation dose-volume effects in the lung. Int J Radiat Oncol Biol Phys 76 (Suppl 3): S70-S76, 2010.

19. Lopez Guerra JL, Gomez D, Zhuang Y, et al: Change in diffusing capacity after radiation as an objective measure for grading radiation pneumonitis in patients treated for non-small-cell lung cancer. Int J Radiat Oncol Biol Phys 83: 1573-1579, 2012.

20. Prevost JB, Voet P, Hoogeman M, et al: Four-dimensional stereotactic radiotherapy for early stage non-small cell lung cancer: a comparative planning study. Technol Cancer Res Treat 7: 27-33, 2008.

21. Gouders D, Maingon P and Paesmans M, et al: Exclusive radiotherapy for non-small cell lung cancer. A retrospective multicentric study. Rep Pract Oncol Radiother (Polish Soc Rad Oncol) 8: 7-14, 2003.

22. Sibley GS, Jamieson TA, Marks LB, et al: Radiotherapy alone for medically inoperable stage I non-small-cell lung cancer: the Duke experience. Int J Radiat Oncol Biol Phys 40: 149-154, 1998

23. Chen VJ, Oermann E, Vahdat S, et al: CyberKnife with tumor tracking: an effective treatment for high-risk surgical patients with stage I non-small cell lung cancer. Front Oncol 2: 9, 2012. 